\title{
Numerical and experimental study of the motion of a sphere in a communicating vessel system subject to sloshing ${ }^{\odot}$
}

Cite as: Phys. Fluids 31, 087106 (2019); https://doi.org/10.1063/1.5098999

Submitted: 05 April 2019 . Accepted: 25 July 2019 . Published Online: 16 August 2019

Esteban Zamora (D), Laura Battaglia (D), Mario Storti (D), Marcela Cruchaga (D), and Roberto Ortega (D)

COLLECTIONS

This paper was selected as Featured
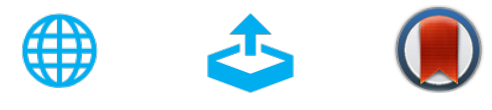

\section{ARTICLES YOU MAY BE INTERESTED IN}

Impact of the Lewis number on finger flame acceleration at the early stage of burning in channels and tubes

Physics of Fluids 31, 083606 (2019); https://doi.org/10.1063/1.5108805

Hydrodynamics of forward blood spattering caused by a bullet of general shape

Physics of Fluids 31, 084103 (2019); https://doi.org/10.1063/1.5111835

Statistical analysis of vortical structures in turbulent boundary layer over directional grooved surface pattern with spanwise heterogeneity

Physics of Fluids 31, 085110 (2019); https://doi.org/10.1063/1.5110048

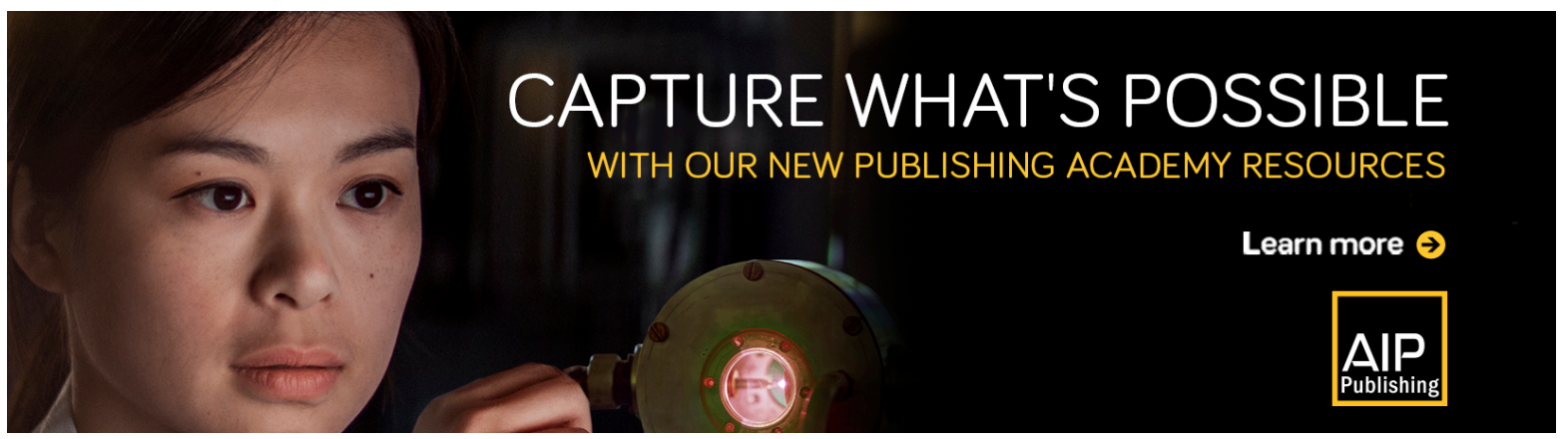




\title{
Numerical and experimental study of the motion of a sphere in a communicating vessel system subject to sloshing ${ }^{\circ}$ ()
}

\author{
Cite as: Phys. Fluids 31, 087106 (2019); doi: 10.1063/1.5098999 \\ Submitted: 5 April 2019 - Accepted: 25 July 2019 • \\ Published Online: 16 August 2019
}

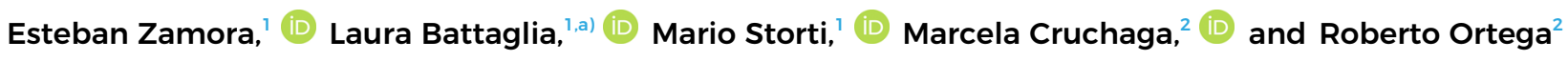

AFFILIATIONS

${ }^{1}$ Centro de Investigación de Métodos Computacionales (CIMEC), Universidad Nacional del Litoral - CONICET, Predio CONICET Santa Fe, Colectora Ruta Nac 168, Km 472, Paraje El Pozo, Santa Fe, Argentina

${ }^{2}$ Departamento de Ingeniería Mecánica, Universidad de Santiago de Chile, Av. Bernardo O’Higgins 3363, Santiago, Chile
\end{abstract}

a) Author to whom correspondence should be addressed: Ibattaglia@cimec.unl.edu.ar

\begin{abstract}
The purpose of this work is twofold: to present a computational strategy to simulate the dynamics of a rigid sphere during water sloshing and to validate the model with original experimental data. The numerical solution is obtained through the coupling between a two-fluid NavierStokes solver and a rigid solid dynamics solver, based on a Newton scheme. A settling sphere case reported in the literature is first analyzed to validate the numerical strategy by ascertaining the settling velocity. In addition, an experiment is carried out based on a sphere submerged into a communicating vessel subjected to sloshing. Experimental data are captured using image processing and statistically treated to provide sphere dynamics quantitative information. The effects of different classical models used to describe drag coefficients, added mass, and wall effects are considered in the study to evaluate their influence on the results. The numerical model provides results that are consistent with the physical data, and the trajectory analysis shows good agreement between the simulations and the experiments.
\end{abstract}

Published under license by AIP Publishing. https://doi.org/10.1063/1.5098999

\section{INTRODUCTION}

Interactions between fluids and rigid solids occur in many engineering applications at different scales, such as in the processing of mineral ores, food processing, ${ }^{1}$ ocean and naval engineering, ${ }^{2,3}$ and renewable energy generators.

The simulation of single or multiple bodies immersed in fluids can be performed via different numerical approaches, where a major issue is how to represent the interface between the body and the surrounding fluid, and their interactions. Some of the following approaches are commonly used: body-fitted and embedded/immersed techniques. Body-fitted schemes, such as the arbitrary Lagrangian-Eulerian techniques, ${ }^{5,6}$ involve discretizing the fluid surrounding the object such that the contour of the body is perfectly represented; however, these methods are not well suited for large displacements because of grid distortion. The embedded and immersed methods represent the contours of objects as nonconforming geometric entities inside the fluid discretization, i.e., the body boundaries cross the faces of the grid. ${ }^{7,8}$ Embedded strategies can be combined with different numerical methods for solving the fluid flow, e.g., lattice Boltzmann methods ${ }^{9-11}$ and Navier-Stokes classic solvers. ${ }^{12-15}$ When a fluidfluid interface or a free surface appears, the flow solver has to be extended, e.g., via a level-set method $^{2,3}$ or a volume-of-fluid approach. ${ }^{16}$

With respect to the sloshing phenomena, different approaches have been developed to predict the interface behavior. Some solutions derived from potential flow theory, where the fluid is assumed to be incompressible and inviscid, have been obtained and applied to particular tank shapes, filling levels, and excitation directions. ${ }^{17-19}$ Other approaches are based on numerical methods for solving viscous free surface and two-fluid flow numerical methods ${ }^{20,21}$ and have been successfully applied to sloshing simulations in two- and three-dimensional problems, as supported by the validations performed with analytical and experimental reference values. $^{22-25}$ 
In the present work, a physical experiment that involves a sphere with slightly negative buoyancy immersed in two communicating vessels submitted to sloshing is presented. Furthermore, a numerical strategy is developed to reproduce the experiment, taking into account the fluid flow simulation and the rigid body motion as separate stages.

For the fluid problem, the Navier-Stokes equations for incompressible and viscous fluids are solved within a level set approach for capturing the movement of the interface between two fluids, i.e., water and air, via the Finite Element Method (FEM). Moreover, the rigid solid dynamics is solved with a Newton scheme. One-way coupling is applied to solve these two linked dynamics, i.e., the velocity and pressure fields from the fluid flow are used to compute the forces acting on the body, which are used in the rigid solid equations. The effects of different classical models used to describe drag coefficients, added mass, and wall effects are considered in the study to evaluate their influence on the results.

The main aspects of this work can be summarized as follows: the report of a new experiment with a sphere submerged inside a sloshing system; the proposal of a strategy to systematize the experimental results from image processing; the development of a numerical method with the ability to reproduce the behavior of a sphere immersed in a viscous fluid in motion; and a statistical treatment of the numerical and experimental results.

The remainder of this work is organized as follows. In Sec. II, the physical experiment and the description of the postprocessing of the results are described. Section III summarizes the computational approach for solving the fluid flow and the motion of the rigid solid. The results are presented in Sec. IV, which includes an initial validation of the numerical model with a well-known test of a settling sphere and a comparison between the experimental and numerical results of the sphere under sloshing. Finally, concluding remarks are presented.

\section{EXPERIMENTAL WORK}

The present experiment is designed to validate the proposed methodology to simulate complex phenomena involving free surface, and fluid and rigid body interaction problems.

\section{A. Experimental layout}

An acrylic tank, divided by a vertical middle wall into two internal vessels, is mounted onto a shake table and subjected to controlled oscillating motion with an amplitude of $A=10 \mathrm{~mm}$ and frequency $f=1 \mathrm{~Hz}$ in the $x$-direction. The tank geometry is shown in Fig. 1. The internal vessels are communicating via a lower opening of $60 \mathrm{~mm}$. A hollow sphere with an external diameter $40.5 \pm 0.5 \mathrm{~mm}$ and wall thickness $9.5 \pm 0.5 \mathrm{~mm}$ is made of a polymer, commercially named polylactic acid (PLA), with a density $\rho_{P L A} \approx 1250 \mathrm{~kg} / \mathrm{m}^{3}$. The sphere weighs $w_{s}=35.93 \pm 0.01 \mathrm{~g}$ (experimentally determined), resulting in a density $\rho_{s}=1033 \pm 21 \mathrm{~kg} / \mathrm{m}^{3}$. The sphere is submerged into distilled water inside the tank, i.e., the sphere has nearly $1.01-1.06$ relative density $\left(\rho_{s} / \rho_{f}\right)$ with respect to the water promoting a slightly negative buoyancy effect. The experiments are performed for a filling depth of $200 \mathrm{~mm}$.

The experiments are recorded using a camera with a resolution of $700 \times 500 \mathrm{px}$ at $10 \mathrm{fps}$. The free surface evolution and sphere

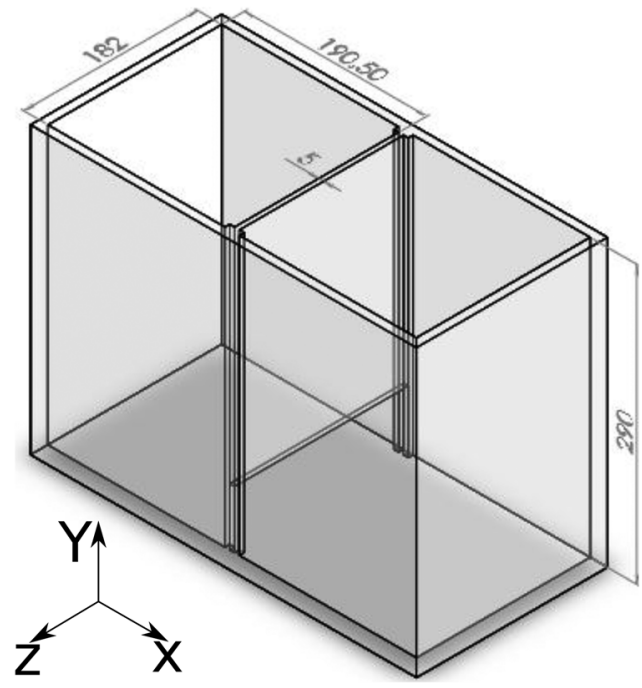

FIG. 1. Geometry of the tank with dimensions in millimeters.

position are measured via postprocessing the videos. The shake table motion and the camera are synchronized. The experimental setup is shown in Fig. 2.

Figure 3 plots a cycle of the shake table motion, including the instants where the frames are taken, i.e., there are 10 frames per cycle. The position of the shake table at the instant labeled as 0 corresponds to the origin of the table displacement; at that moment, the table has null displacement and maximum velocity and is moving from left to right. Instant 5 corresponds to the tank being centered and moving to the left. By symmetry, instant 5 is completely equivalent to instant 0 , with symmetry reflection about the $x$-axis $(x \rightarrow-x)$. In the following, when the statistical results are presented for instant 0 , they also include the results for instant 5 after applying the appropriate symmetry. This symmetry is kinematically valid only for the time periodic regime. On the basis of this property, the number of relevant frames is reduced by one-half and the data are increased twofold. In the same way, instant 1 is equivalent to instant 6 and so on.

\section{B. Image capturing technique}

The instantaneous positions of the sphere are obtained via an image processing technique. To this end, an in-house code was developed in C++ using OpenCV ${ }^{26,27}$ and Armadillo ${ }^{28,29}$ as a library for sparse matrix linear algebra.

The center of the sphere is determined by means of image processing. The procedure is based on histogram and thresholding techniques, ${ }^{30,31}$ where a region of interest (ROI) is defined, i.e., the green rectangle centered at the spheres shown in Figs. 4 and 8. Initially, the ROI is characterized by a histogram in HSV color space (represented by channels: hue, saturation, value). To identify the sphere, the variation in color in the hue channel is considered because the sphere has only one color, i.e., the saturation does not significantly change, and the lighting is sufficient to maintain a uniform value, i.e., the hue values remain constant under slight variation in light. Then, to 


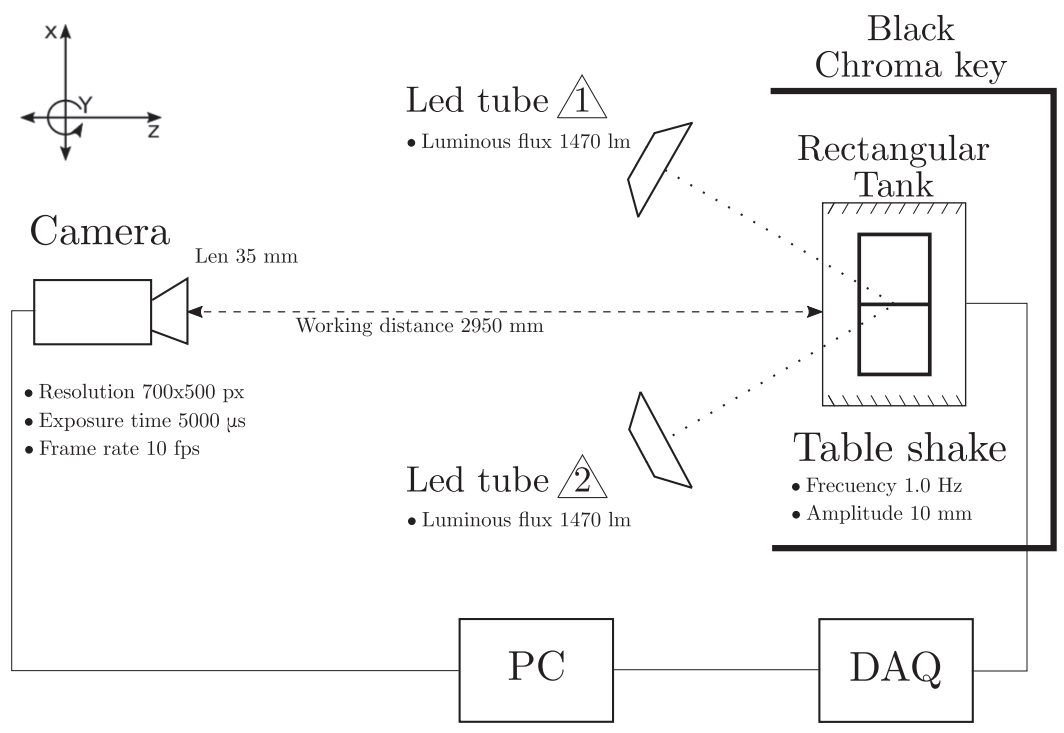

FIG. 2. Experimental setup.

separate the sphere from the background, a binary mask is applied to the search zone identified by the red rectangle in Figs. 4 and 8, where the range of hue values is used as belonging criteria. Finally, to obtain the coordinates of the center of the sphere, the Hough circle transform function implemented in the OpenCV library is applied. This function uses the Hough gradient method and the gradient information of the contours from binary mask, for each frame. The next searching zone in the following frame is defined as a square, where the side length is equal to twice the diameter of the sphere and center is coincident with the current coordinates of the center of the sphere. The results are saved in a tracking table that contains all the positions for each frame, and a history map is constructed to store the number of times that the center of the sphere passed through a certain pixel of the domain.

The main uncertainties in the sphere location come from the diameter measure due to the distance to the focal plane. In order to assess the precision of the algorithm used in this work, a simple test

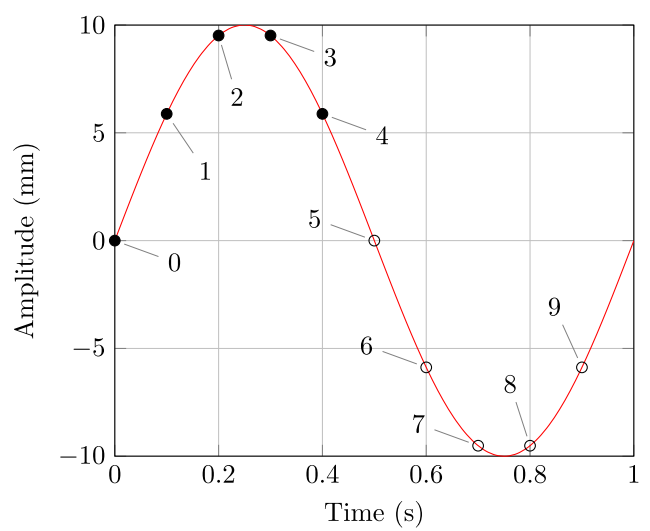

FIG. 3. Classification of the frames by their position relative to the cycle. was carried out. For this, 1000 random spheres have been generated inside a bitmap of $100 \times 100 \mathrm{px}$, where the diameter is randomly disturbed to emulate the uncertainty to the focal plane in the experiments. The diameter used in this analysis was of 67 px. Figure 4 shows some samples of the random positions and sizes of the sphere in an unfavorable situation, where the white circle is the detected sphere. Note that, for a fixed diameter, smaller or larger gray zones represent a sphere behind or ahead of the focal plane, respectively. From this analysis, the precision of the algorithm to determine the center of a sphere is $\pm 2 \mathrm{px}$. This error represents $1.2 \mathrm{~mm}$ in the experiment.

\section{Experimental methodology}

The experimental data are obtained from 18 experiments conducted during $766.26 \mathrm{~min}$ of the free surface time periodic regime (equivalent to 459770 frames). The data are presented in Sec. IV together with the results computed from the simulations.

The sphere is randomly released at the bottom of a vessel. To evaluate the results of the sphere position over time obtained from the image capturing technique, parameters of the sphere (trajectories) are measured, and statistically reproducible quantities, such as the location and velocity, are calculated.

A classification based on where the sphere is initially located is used to describe trajectories. The left vessel is divided into 4 regions, numbered clockwise from the top right. The right vessel is also divided into 4 regions, numbered counterclockwise from the top left (specular counterpart of the left vessel).

Regarding the spatial distribution, $x$ - (or $y$-) axis is divided into 6 bins, and the number of times that the sphere is in each bin is computed.

The mean velocity of the sphere is computed on the basis of the difference in positions at subsequent frames and the time steps between these frames. This velocity is taken as a reference for 

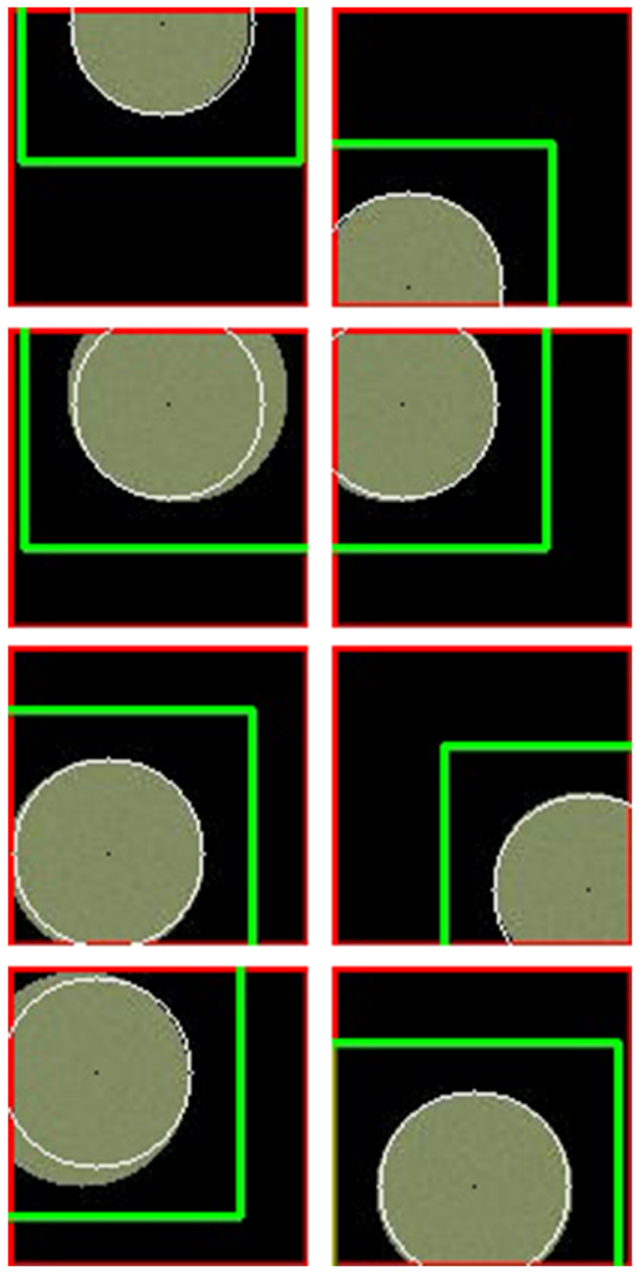

FIG. 4. Image capturing technique uncertainty assessment. Some random sphere positions, search zones, and results obtained from the algorithm.

comparison with the numerical results in Sec. IV B, particularly with the average velocity and a histogram.

Moreover, the free surface is tracked over time at certain control points by means of image processing, and these measurements are considered to contrast the fluid dynamics results.

Some overall remarks can be noted from the videos. The sphere seldom impacts the vessels walls, the sphere never emerges from the water, and the sphere rarely crosses from one vessel to the other through the opening. These facts are confirmed in the simulations.

It is also remarkable that no high rotation has been observed during the analysis of this highly transient problem. Due to this fact, only translation motion was considered in the present study. To confirm this assumption, the sphere rotation has been estimated from the images, and a value of $1.1 \mathrm{rad} / \mathrm{s}$ has been found with a low recurrence. This rotation was used to compute lift Magnus forces, and they are small enough to not take rotation into account as will be declared in Secs. III and IV B.

\section{MATHEMATICAL MODEL}

\section{A. Fluid dynamics formulation and free surface description}

The two-fluid flow is described by the Navier-Stokes equations for viscous incompressible flow,

$$
\begin{aligned}
& \rho\left(\frac{\partial \mathbf{v}}{\partial t}+\mathbf{v} \cdot \nabla \mathbf{v}-\mathbf{f}\right)-\nabla \cdot \boldsymbol{\sigma}=0 \quad \text { in } \Omega, \\
& \nabla \cdot \mathbf{v}=0 \quad \text { in } \Omega \text {, }
\end{aligned}
$$

where $\rho$ is the density, $\mathbf{v}$ is the fluid velocity, and $\mathbf{f}$ represents the external body forces per unit mass, over the spatial domain $\Omega$ in time $\mathrm{t} \in[0, \mathrm{~T}]$. Furthermore, the stress tensor $\boldsymbol{\sigma}$ in Eq. (1) is defined as $\boldsymbol{\sigma}=-p \mathbf{I}+\mu\left(\nabla \mathbf{v}+\nabla \mathbf{v}^{T}\right)$, where the first term is the isotropic part, with pressure $p$ and identity tensor $\mathbf{I}$. The second term is the deviatoric part, i.e., the viscous stress tensor for a Newtonian fluid, where $\mu$ is the dynamic viscosity of the fluid. The fluid properties are distributed in the domain according to the free surface presence, as indicated below.

The system of Eq. (1) is solved by a finite element strategy with finite elements of equal order for velocity and pressure, stabilized with streamline upwind/Petrov-Galerkin (SUPG) ${ }^{33}$ and pressure-stabilizing/Petrov-Galerkin (PSPG).

Several works conclude that turbulent dissipation is an important damping factor in the sloshing physics, ${ }^{35}$ as well as in other free surface flows. ${ }^{36}$ Hence, a simple turbulence model is used to consider some viscous dissipative effects such that the dynamic viscosity is replaced by $\mu_{t}=\min \left(\mu+C_{s}^{2} h_{s}^{2} \rho \sqrt{2 \epsilon: \epsilon}, \mu_{\max }\right)$, where $C_{s}$ is the Smagorinsky coefficient, $\epsilon=\frac{1}{2}\left(\nabla \mathbf{v}+\nabla \mathbf{v}^{T}\right)$ is the strain rate, $h_{s}$ is the characteristic length of an element, and $\mu_{\max }$ is a cutoff value. This model has been applied before for solving more challenging sloshing cases, as in Refs. 21 and 24.

The free surface is modeled by an interface capturing technique that relies on a continuous and smooth level set like function $\phi$. The level set transport, represented by

$$
\frac{\partial \phi}{\partial t}+\mathbf{v} \cdot \nabla \phi=0 \quad \text { in } \quad \Omega
$$

requires the velocity field $\mathbf{v}$ from Eq. (1) to advance in time. These equations are also solved by SUPG stabilized finite elements, over the same mesh as the fluid flow problem.

As Eq. (2) does not preserve certain properties of the $\phi$ field, such as the transition profile from one to another fluid, a numerical procedure is applied to regularize the field, which consists of solving

$$
\phi\left(\phi^{2}-\phi_{\text {ref }}^{2}\right)-\kappa \Delta \phi+M\left(\hat{H}(\phi)-\hat{H}\left(\phi_{0}\right)\right)=0 \quad \text { in } \Omega,
$$

over the whole domain $\Omega$, where $\phi_{0}$ is obtained from Eq. (2), the reference value is $\phi_{\text {ref }}=1, \kappa$ is the diffusivity, $M$ is a penalty coefficient, and the expression for the penalizing term is $\hat{H}(\phi)=\tanh (2 \pi \phi)$. This third problem is also solved with a finite element method, which is steady in each time step ${ }^{38}$ and can be complemented with a global explicit volume preservation stage. ${ }^{2}$

Hence, the properties of the fluids are distributed in the whole domain according to the marker function $\phi(\mathbf{x}, t)$ that is positive in the liquid part of the domain, $\Omega_{l}$, negative in the gaseous phase, $\Omega_{g}$, and is null specifically over the free surface $\Gamma_{\mathrm{FS}}$ such that $\Omega=\Omega_{l} \cup \Omega_{g}$ and $\Omega_{l} \cap \Omega_{g}=\emptyset$. Particularly, and due to the renormalization stage 
applied, it is $-1<\phi<1 .^{38}$ Then, the density and the viscosity are given as functions of $\phi$, with

$$
\eta(\phi)=\frac{1}{2}\left[(1+\tilde{H}(\phi)) \eta_{l}+(1-\tilde{H}(\phi)) \eta_{g}\right],
$$

where $\eta(\phi)$ represents either $\rho(\phi)$ or $\mu(\phi)$, and subscripts $l$ and $g$ indicate the liquid or the gaseous phase, respectively. In the present work, $\tilde{H}(\phi)=\tanh \left(\frac{\pi \phi}{\tilde{\varepsilon}}\right)$ such that it is a smooth sign function, where the parameter $\tilde{\varepsilon}$ calibrates the width of the transition of the properties between the fluids.

\section{B. Solid-rigid dynamics}

The expression for the solid-rigid dynamics corresponds to the application of Newton's second law for displacement,

$$
\mathbf{m}_{\text {sph }} \ddot{\mathbf{q}}+\mathbf{F}_{\mathrm{c}}=\mathbf{F}_{\text {ine }}+\mathbf{F}_{\mathrm{fl}},
$$

where the first term corresponds to inertial forces, $\mathbf{m}_{\mathrm{sph}}$ is the mass matrix corresponding to the body, $\mathbf{q} \in \mathbb{R}^{n}$ is the displacement vector in $n$ coordinates, and $\ddot{\mathbf{q}}$ is the corresponding acceleration vector. The second term, $\mathbf{F}_{\mathbf{c}}$, includes the internal forces representing the rigid contact constraints, and the right-hand side quantifies the external forces acting on the center of the solid, considered as a particle. The external forces considered in the present work are due to the accelerations applied to the system and the fluid forces over the sphere.

The rigid-contact force that appears on the sphere due to the vessel walls is modeled by a penalty method. The contact forces are

$$
\mathbf{F}_{\mathbf{c}}=k_{c} d \mathbf{n}+\left\langle\beta_{c} \dot{\mathbf{q}} \mathbf{n}\right\rangle_{\delta},
$$

where the first term accounts for the contact forces as a function of the signed distance function $d=d(\mathbf{q})$ between the sphere and the vessel walls such that, when $d \geq 0$, the sphere does not contact the wall and there are no contact forces acting over the sphere. When $d<0$, there is penetration and, consequently, the contact force magnitude is determined as $k_{c} d$, where $k_{c}$ is the penalty coefficient and $\mathbf{n}$ is the unit vector normal to the interface, $\mathbf{n} \approx \frac{\nabla d}{\|\nabla d\|}$. The second term considers the approximation effect near the walls; ${ }^{39,40}$ this force is activated at a distance equal to $\delta$ of the radius of the sphere, expressed as a percentage of the radius of the sphere, where $\beta_{c}$ is a constant coefficient. A more sophisticated mechanism in order to consider the lubrication effects when the sphere is very close to the wall could be developed. These models require a proper correlation with Re and wall distance. ${ }^{41,42}$ Other authors quantify the wall influence as a correction on the settling velocity ${ }^{13,43}$ or wall influence at a low Re number ${ }^{44,45}$ as examples.

The inertial forces due to nongravitational accelerations $\mathbf{g}_{\text {box }}$ applied over the noninertial reference frame are determined as $\mathbf{F}_{\text {ine }}=\mathbf{m}_{\text {sph }} \mathbf{g}_{\text {box }}$.

Furthermore, the fluid forces comprise the buoyancy forces $\mathbf{F}_{\text {buoy, }}$, the drag forces $\mathbf{F}_{\text {drag }}$, and the added mass forces $\mathbf{F}_{\text {add }}$, i.e., $\mathbf{F}_{\text {fl }}=\mathbf{F}_{\text {buoy }}+\mathbf{F}_{\text {add }}+\mathbf{F}_{\text {drag }}$.

The buoyancy term is $\mathbf{F}_{\text {buoy }}=-V \nabla p$, where $V$ is the volume of the sphere. Note that this term considers the full lift forces due to the pressure gradient from the fluid state. Other lift forces, such as Magnus and Saffman forces, are not included in the model. The Magnus force evolves during imposed rotation in the rigid body. ${ }^{46,47}$ Saffman forces $^{48,49}$ are developed under high velocity gradients and are commonly applied to small to nanoparticle analyses. In the present analysis, as will be shown in Sec. IV B, these forces are negligible in comparison with the lift due to the pressure gradient.

The added mass force is defined as

$$
\mathbf{F}_{\mathrm{add}}=-m_{\mathrm{add}}\left(\ddot{\mathbf{q}}+\mathbf{g}_{\mathrm{box}}\right),
$$

with the added mass $m_{\text {add }}=k_{\text {add }} \rho V$, where $\rho$ is the fluid density. The added mass coefficient is determined as a function of the Reynolds number $\left(\operatorname{Re}=\rho\left|\mathbf{v}_{r}\right| D_{s} \mu^{-1}\right),{ }^{50,51}$

$$
k_{\text {add }}=\left\{\begin{array}{ll}
8.591 \operatorname{Re}^{-0.5835} & \text { if } \operatorname{Re} \leq 100 \\
15 \operatorname{Re}^{-1.125}+0.5 & \text { if } \operatorname{Re}>100
\end{array} .\right.
$$

The drag forces are modeled as

$$
\mathbf{F}_{\text {drag }}=-\frac{1}{2} C_{D} A_{s} \operatorname{Re} \frac{\mu}{D_{s}} \mathbf{v}_{r},
$$

where the drag coefficient $C_{D}$ is determined as a function of Re, the relative velocity between the sphere and the flow is $\mathbf{v}_{r}=\dot{\mathbf{q}}-\mathbf{v}, D_{s}$ is the sphere diameter, and $A_{s}$ is the reference area, in this case the projected area of the sphere. Different expressions for the drag coefficient can be found in the literature based on a sphere immersed in flows at constant relative velocities, i.e., the sphere moves with constant velocity with respect to the fluid. An empirical relationship covering a wide range of Re is proposed in Ref. 52, from which the following expression is adopted:

$$
C_{D}=\left(\varphi_{1}+\varphi_{2}\right)^{1 / 10}
$$

This equation is valid for the Re range of the analyses presented in this work, i.e., $\operatorname{Re}<2 \times 10^{5}$, where $\varphi_{1}=\left(\frac{24}{\operatorname{Re}}\right)^{10}+\left(\frac{21}{\operatorname{Re}^{0.67}}\right)^{10}+\left(\frac{4}{\operatorname{Re}^{0.33}}\right)^{10}$ $+(0.4)^{10}$ and $\varphi_{2}=\left[\left(0.148 \mathrm{Re}^{0.11}\right)^{-10}+0.5^{-10}\right]^{-1}$. A heuristic relation is proposed in Ref. 53 based on boundary layer theory,

$$
C_{D}=\frac{24}{(9.06)^{2}}\left[1+\frac{9.06}{\sqrt{\mathrm{Re}}}\right]^{2} .
$$

Furthermore, the following expression used in Ref. 13 is also applied:

$$
C_{D}=a \mathrm{Re}^{-b},
$$

where $a$ and $b$ are adjusted by fitting the experimental drag values reported in literature. ${ }^{52,54}$

\section{Solver coupling}

The numerical strategy is proposed as a one-way coupling, where the fluid forces over the sphere are modeled by taking as data the fluid state, determined from Eq. (1), and the forces due to the added mass phenomenon.

The procedure is sketched in Fig. 5. The velocity $\mathbf{v}^{t_{f}}$ and the pressure $p^{t_{f}}$ are known from the fluid state at time $t_{f}$ in every node of the corresponding mesh. Then, the velocity at the center of the sphere, and in certain cases at other points over the circumference, is calculated via FEM interpolation, giving the mean velocity $\mathbf{v}_{f}^{t_{f}}$ for the body. The relative velocity of the solid $\mathbf{v}_{r}^{t_{s}}$ at the solid time step $t_{s}$ is determined as

$$
\mathbf{v}_{r}^{t_{s}}=\dot{\mathbf{q}}^{t_{s}}-\mathbf{v}_{f}^{t_{f}} .
$$




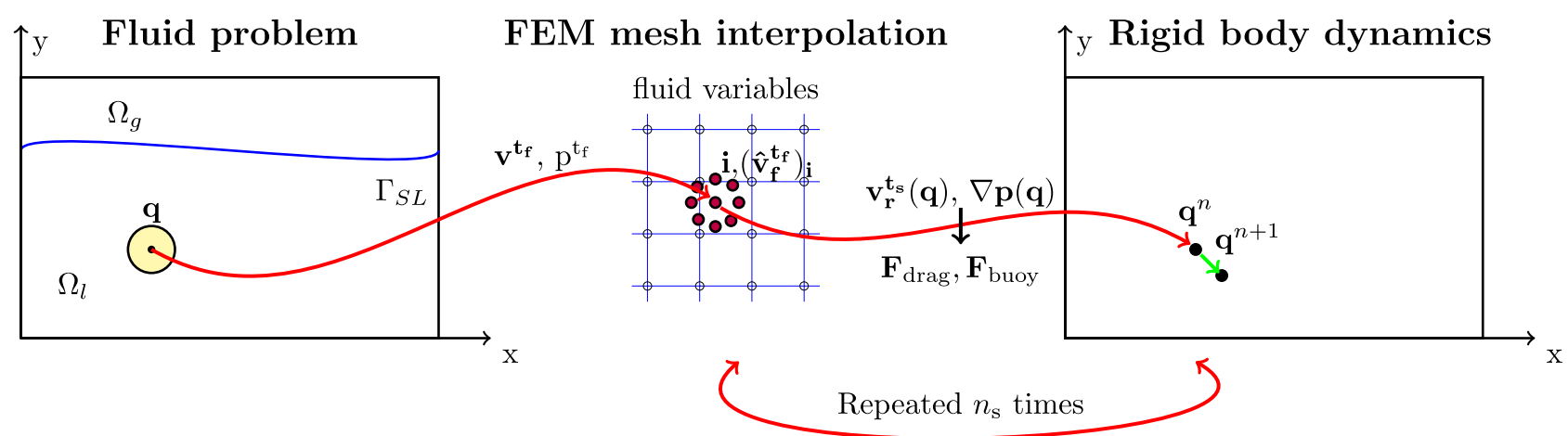

FIG. 5. Data exchange between the fluid and the solid dynamics involved in the algorithm.

The relative velocity of the sphere with respect to the fluid is employed to determine $\mathbf{F}_{\mathrm{drag}}$, while the pressure gradient is considered in $\mathbf{F}_{\text {buoy }}$. Note that the time step of the solid is several times smaller than the fluid time step. Then, the interpolation of the velocity and the pressure is repeated $n_{s}$ times for each fluid time step, with $n_{s}=\Delta t_{f} / \Delta t_{s}$.

\section{NUMERICAL RESULTS}

Two cases are studied to further validate the reliability of the model. The first case corresponds to the settling of a single sphere, and the other corresponds to sloshing in a communicating vessel problem described in Sec. II.

\section{A. Settling of a single sphere}

Several authors appeal to the settling of a sphere as an initial validation for their fluid-structure interaction numerical schemes. ${ }^{9-11,13,55,56}$ Following the analysis presented by Ref. 11, the settling of a spherical particle of radius $7.5 \mathrm{~mm}\left(r_{p}\right)$ and density $\rho_{s}=1250 \mathrm{~kg} / \mathrm{m}^{3}$ is studied. Initially, both the fluid and the particle are at rest, and the particle is freely released under gravity from an initial position of $(0.0,0.0,127.5) \mathrm{mm}$ in a rectangular container of size $[-50,50] \times[-50,50] \times[0,160] \mathrm{mm}^{3}$. A schematic diagram of the settling case is depicted in Fig. 6 . The motion is induced by gravity and is developed due to the hydrostatic pressure gradient. Three different cases are considered by varying the properties of the fluid, as shown in Table I.

Table II summarizes the time step $\Delta t_{s}$ for the solid stage, the parameters $\beta_{c}$ and $\delta$ for the contact forces in Eq. (6), and the drag law and parameters $a, b$ used for the corresponding drag law in each case, if needed.

Figure 7(a) shows the evolution of the vertical position of the sphere until it reaches the bottom, and Fig. 7(b) plots the velocity of the sphere. Three stages are observed during the settling of a sphere: when the sphere is free falling (acceleration stage), when the sphere reaches terminal velocity (constant velocity stage), and, finally, when the sphere approaches the bottom (deceleration stage). The present model agrees with the experimental data in these three stages. The experimental absolute error reported by the reference is lower than 0.1 in the relative position $h^{*}$. The added mass effect has a greater influence in the first stage because $k_{a d d}$ is inversely proportional to
Re. The drag force given via Eq. (12) fits better for Re 4.1 and 11.6, while for Re 32.2, Eq. (10) fits better. The approximation effects near the bottom of the rectangular container are clearly appreciated in the low $\operatorname{Re}$ case $(\operatorname{Re} 4.1)$. Parameter $\beta_{c}$ and its activating zone $\delta$ are chosen to reproduce the deceleration stage. Particularly, both parameters have been increased to obtain a better approximation for the lower Re case.

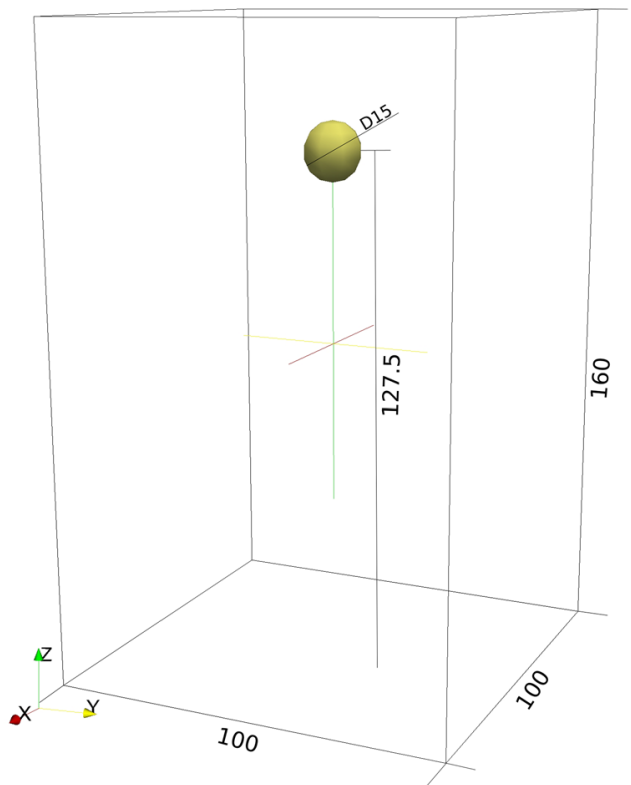

FIG. 6. Single particle settling in a rectangular box. Dimensions in millimeters.

TABLE I. Fluid properties for three different Re numbers in the settling of a sphere.

\begin{tabular}{lccr}
\hline \hline Case & $\rho_{f}\left(\mathrm{~kg} \mathrm{~m}^{-3}\right)$ & $\mu_{f}\left(\mathrm{~N} \mathrm{~s} \mathrm{~m}^{-2}\right)$ & $\mathrm{Re}$ \\
\hline 1 & 965 & 212 & 4.1 \\
2 & 962 & 113 & 11.6 \\
3 & 960 & 58 & 32.2 \\
\hline \hline
\end{tabular}


TABLE II. Near-wall settings and drag parameters for the settling of a sphere. Parameters $a, b$ are given for the drag law of Eq. (12).

\begin{tabular}{lccccc}
\hline \hline $\operatorname{Re}$ & $\Delta t_{s}(\mathrm{~s})$ & $\beta_{c}\left(\mathrm{~N} \mathrm{~s} \mathrm{~m}^{-1}\right)$ & $\delta(\%)$ & Drag law & $a ; b$ \\
\hline 4.1 & 0.01 & 160 & 45 & Eq. (12) & $28.01 ; 0.7641$ \\
11.6 & 0.01 & 250 & 20 & Eq. (12) & $27.327 ; 0.7641$ \\
32.2 & 0.005 & 250 & 20 & Eq. (10) & $\ldots$ \\
\hline \hline
\end{tabular}

\section{B. Modeling the experiments}

This section reports the numerical results for the simulation of the experiment described in Sec. II using the methodology reported in Sec. III in comparison with the experimental data. The fluid field is simulated for a certain number of cycles of the imposed shake table motion until the periodic regime is reached. The sphere is injected at a random position and time at instant 0 of the cycle, with the instantaneous fluid velocity at that point and instant. Then, the sphere is tracked using the solid rigid dynamic equations described in Sec. III. For a fair comparison of the results, the statistical analysis requires

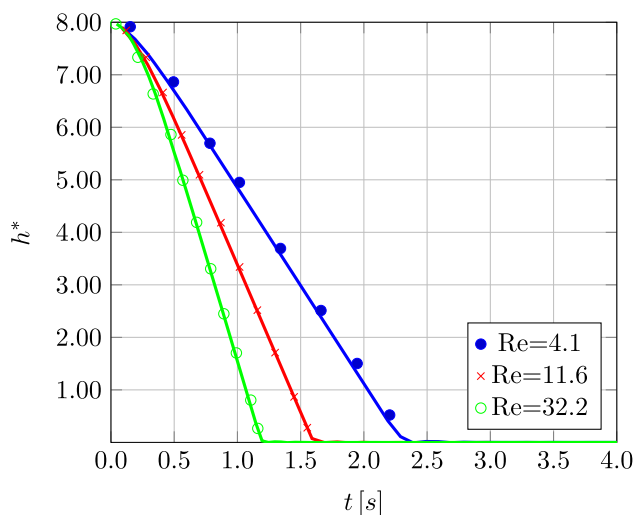

(a)

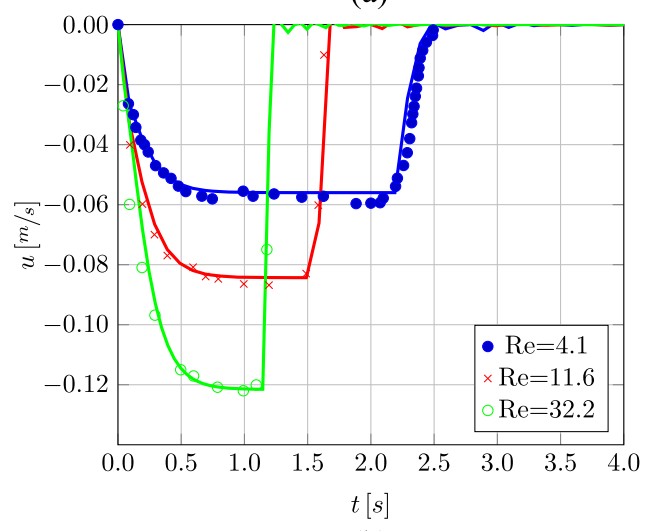

(b)

FIG. 7. Comparison of the experimental data (dots) and simulation results (continuous lines) in terms of (a) vertical position $h^{*}=\frac{z-r_{p}}{2 r_{p}}$ and (b) velocity for different Reynolds numbers. an amount of sphere position data similar to that obtained from the experiments, i.e., a total of 200 simulations of 100 s were performed. These data represent $43.5 \%$ of the experimentally obtained sphere position information. The sphere density $\left(\rho_{s}\right)$ used in the simulations is $1014.92 \mathrm{~kg} \mathrm{~m}^{-3}$. A sensitivity analysis (not shown) of the numerical results with respect to the sphere density was also conducted to evaluate the effect of the density variation. Note that, as the relative density of the sphere with respect to the distilled water is $\approx 1.02$, the sphere has a nearly unit buoyancy and the system is highly sensitive to any slight change in such a property. From the referred analysis, the reported density, chosen within the experimental range, provides the best adjustment between the experimental and numerical results. The contact stiffness coefficient $\left(k_{c}\right)$ is $142950 \mathrm{~N} \mathrm{~m}^{-1}$, which was validated by a mass point contacting plane test, as reported in Ref. 57. The effects of the vicinity to the walls are considered with $\beta_{c}=0.75 \mathrm{~N} \mathrm{~s} \mathrm{~m}^{-1}$ and $\delta=20 \%$. Different runs were analyzed to evaluate the sensitivity of the results to the model parameters. From such numerical tests (not shown), minimal maximum error was found in the analysis of the results with the reported values. Focusing the analysis on $\beta_{c}$, two overall aspects could be remarked. When a sphere approaches a wall at low velocity (i.e., low Reynolds number), not only the wall effect is observed at larger distances from the wall but also the $\beta_{c}$ parameter has to be increased with respect to high Reynolds numbers (see Sec. IV A). Moreover, the results of this section present small changes for $\beta_{c}$ values varying in the range $[0,1] \mathrm{N} \mathrm{s} \mathrm{m}^{-1}$. To complete the sensitivity analysis of the parameters involved in the model present in Sec. III, two different drag laws given by Eqs. (10) and (11) are used, and the models are named set 1 and set 2 , respectively. The time steps for the fluid $\left(\Delta t_{f}\right)$ and the solid solver $\left(\Delta t_{s}\right)$ are set to $0.01 \mathrm{~s}$ and $0.0004 \mathrm{~s}$, respectively, and the sloshing problem was solved with 4 -node finite elements for the following three instances: fluid flow, level set advection, and renormalization. ${ }^{37}$ The properties of the fluids used in this case are shown in Table III, while the parameters for the turbulence model are $C_{s}=1$ and $\mu_{\max }=0.1 \mathrm{~kg}(\mathrm{~ms})^{-1}$, set from previous numerical analyses in sloshing problems. ${ }^{21,24}$

Figure 8 presents snapshots from the video and a simulation at different instants of the free surface periodic regime, in which the center of the sphere follows trajectory 3-4 in Fig. 10(a). The snapshots from the simulations also show the velocity vectors and the free surface position. The free surface evolution is verified first. The free surface displacement, measured as the difference $h_{\text {diff }}$ between the interface measured at CP1 and CP2, is presented in Fig. 9(a), together with the box displacement $x_{\text {box }}$ when the time periodic steady stage is reached; see Fig. 9(b) for reference. The maximum free surface difference is $A_{h_{\text {diff }}} \approx 30 \mathrm{~mm}$,

TABLE III. Properties of the fluids involved in the case of sloshing with a submerged sphere.

\begin{tabular}{lcc}
\hline \hline Property & Symbol & Value \\
\hline Water density & $\rho_{f}\left(\mathrm{~kg} \mathrm{~m}^{-3}\right)$ & 998.2 \\
Water viscosity & $\mu_{f}\left(\mathrm{~N} \mathrm{~s} \mathrm{~m}^{-2}\right)$ & 0.001 \\
Air density & $\rho_{l}\left(\mathrm{~kg} \mathrm{~m}^{-3}\right)$ & 1.225 \\
Air viscosity & $\mu_{l}\left(\mathrm{~N} \mathrm{~s} \mathrm{~m}^{-2}\right)$ & 0.000018 \\
\hline \hline
\end{tabular}



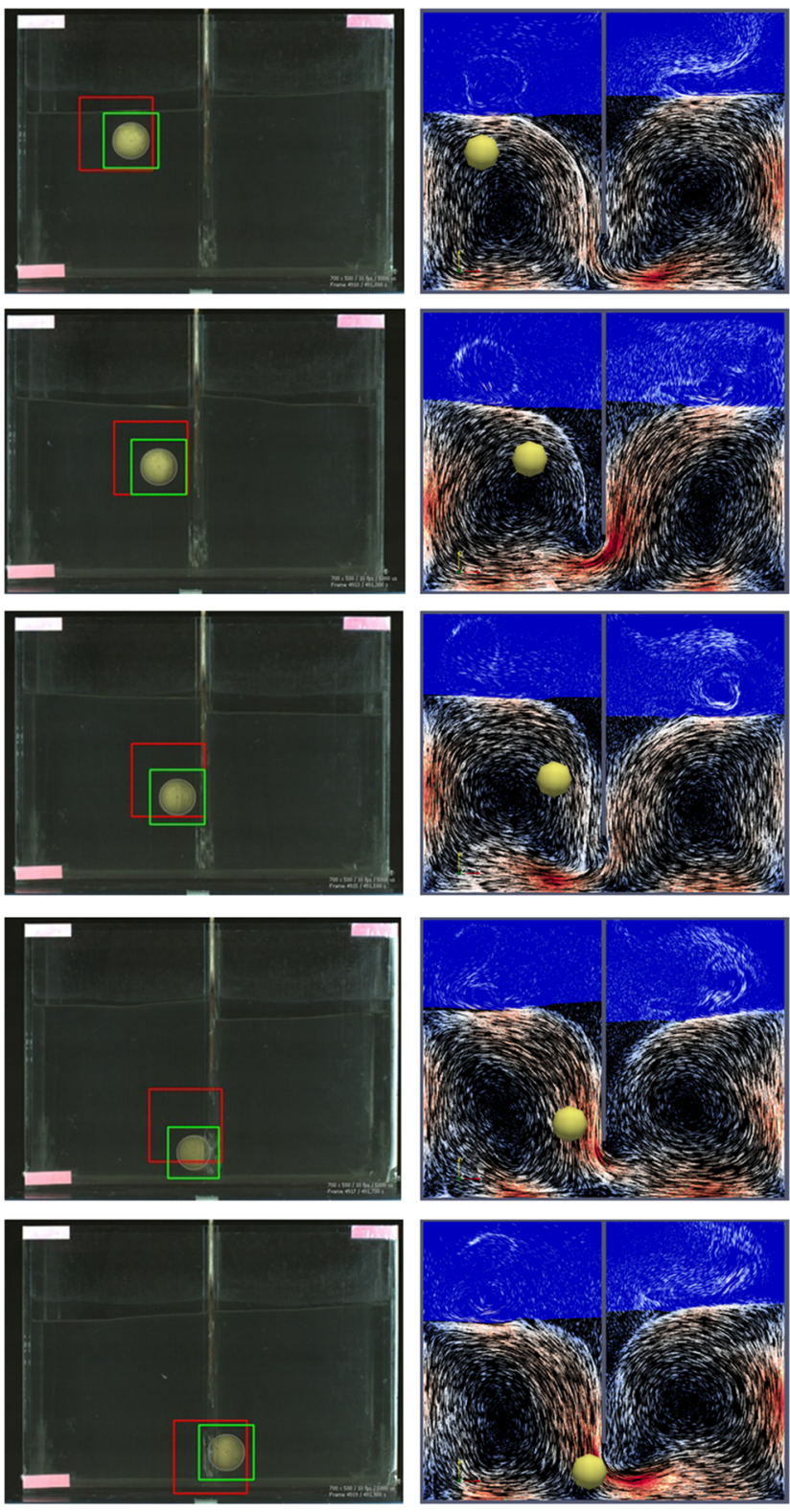

FIG. 8. Some frames corresponding to a cycle during steady state periodic regime at the times shown: $0,3,5,7$, and 9 according to Fig. 3. Experiment (left column) and simulation (right column).

while the imposed maximum displacement is $A_{b o x}=10 \mathrm{~mm}$. Good agreement is observed between the experimental (dots) and numerical results (line), in terms of both amplitude and phase. Furthermore, this agreement is verified by the Lissajous pattern in Fig. 9(b), which compares the experimental (crosses) and numerical (line) measurements.

Figure 10 shows different trajectories observed in the experimental and numerical results. The trajectories correspond to an elapsed time of $3 \mathrm{~s}$. In each figure, the trajectories start in one of

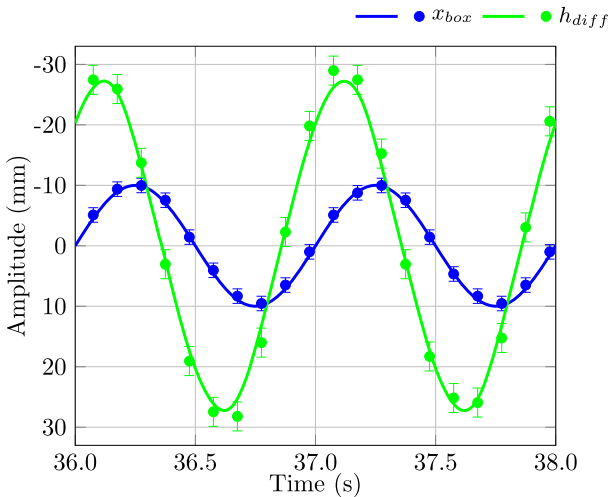

(a)

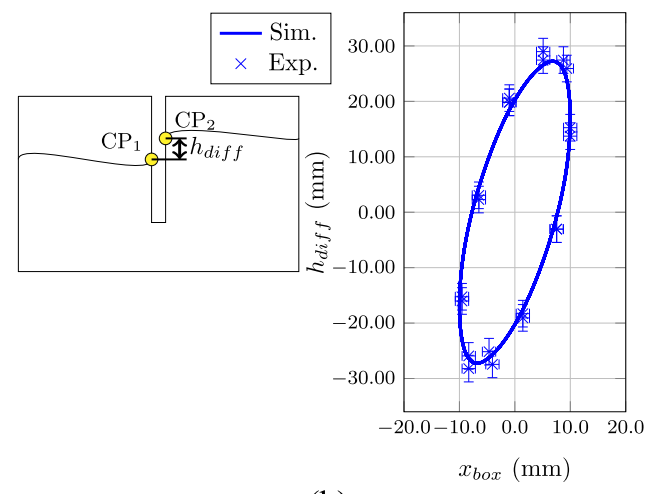

(b)

FIG. 9. Free surface experimental and simulation results. (a) Differences in height $h_{\text {diff }}$ between control points $\mathrm{CP}_{1}$ and $\mathrm{CP}_{2}$ and box displacements $x_{\text {box }}$ for the experimental (dots) and numerical (lines) results. (b) Lissajous pattern with the experimental (crosses) and simulation (line) results.

the four regions of the left vessel and cross from the left to the right vessel. The continuous lines are numerical trajectories, and the dots are the experimental trajectories. Each dot corresponds to a frame. In each figure, the trajectories start in similar initial positions and at equivalent instants relative to the cycle as presented in Fig. 3. As the behavior of the sphere is chaotic and the initial positions and velocities are not equal, the trajectories should not necessarily match; nevertheless, the trajectories are very similar.

Figure 11(a) shows the temporal evolution of the Reynolds number $\operatorname{Re}(t)$, based on the relative velocity of the sphere with respect to the fluid. Three randomly chosen independent simulations (of a total of 200) are shown, labeled as S1, S2, and S3. For each simulation, the trajectory of the sphere is computed for $200 \mathrm{~s}$, but only the central interval from 50 to $150 \mathrm{~s}$ is analyzed. The average Re for each of the simulations is $\bar{R}_{S 1}=3783.3, \overline{R e}_{S 2}=3834.4$, $\bar{R} e_{S 3}=3584.1$. Figure 11(b) shows the spectral analysis (discrete Fourier transform) of $\operatorname{Re}(t)$. The behavior of the three evolutions is similar. In all cases, large amplitudes are observed in the ranges $0.25 \mathrm{~Hz}-0.5 \mathrm{~Hz}$ and $1 \mathrm{~Hz}-1.5 \mathrm{~Hz}$.

In the following, all the histograms show the probability $\mathrm{P}[\%]$ of the determined number of successes over the total number of observations, e.g., in Fig. 12, the number of times that the Re, the 


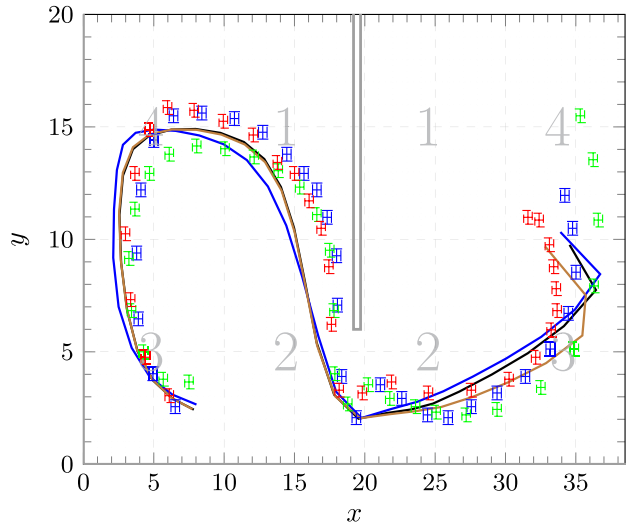

(a)

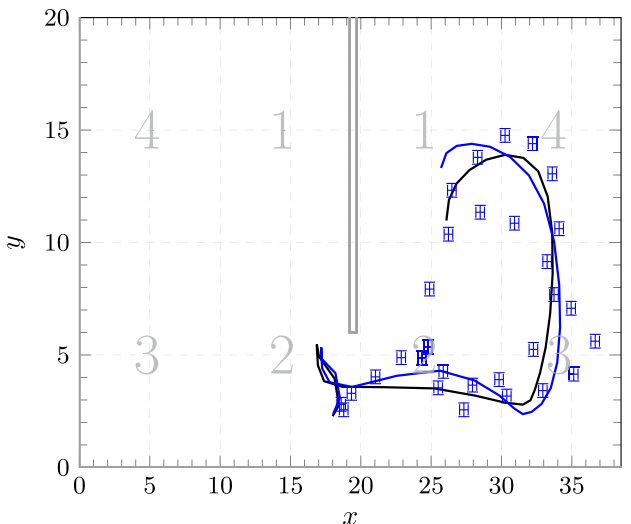

(c)

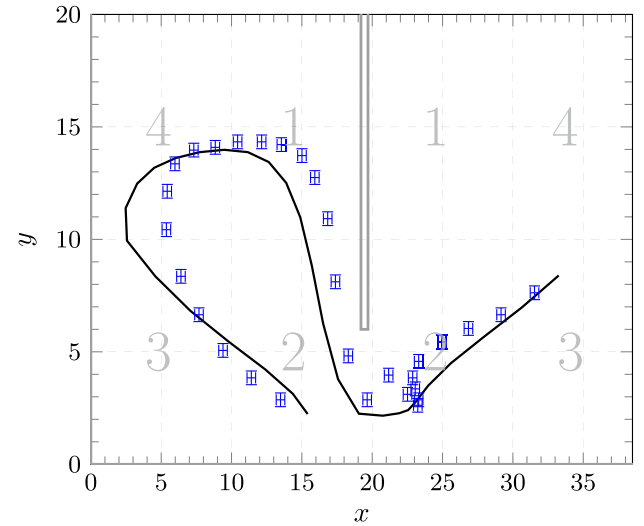

(b)

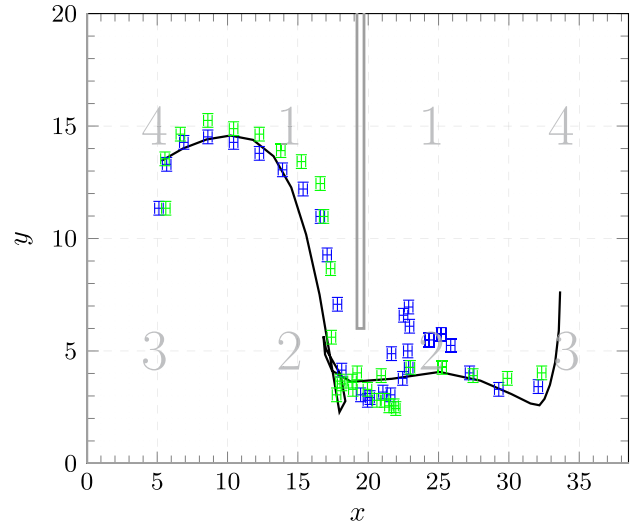

(d)
FIG. 10. Four different types of trajectories of the sphere starting in $\mathrm{i}$ and finishing in j. (a) Trajectory 3-4. (b) Trajectory 2-3. (c) Trajectory 2-1. (d) Trajectory 4-3.
$C_{D}$, or the mean velocity of the sphere $\mathrm{V}_{s}$ is found in a certain range of values over the total number of recorded observations. Figure 12(a) shows the statistical distribution of the Re number found in all simulations for data set 1 and set 2 corresponding to the drag law described in Eqs. (10) and (11), respectively.
The histogram is built by dividing the interval between the minimum and the maximum observed Reynolds into ten bins. According to the numerical results, the Reynolds number ranges from 7.5 to 17527 , and $37 \%$ of the time, it is in the range of $1760-3512$, centered at 2636. This fact modified the drag coefficient accordingly.

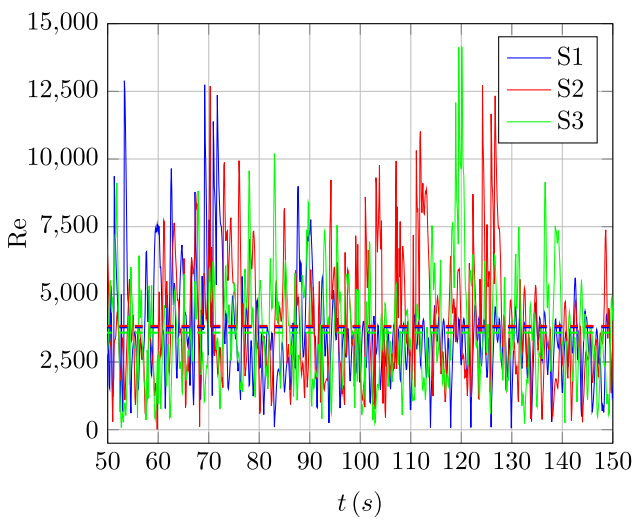

(a)

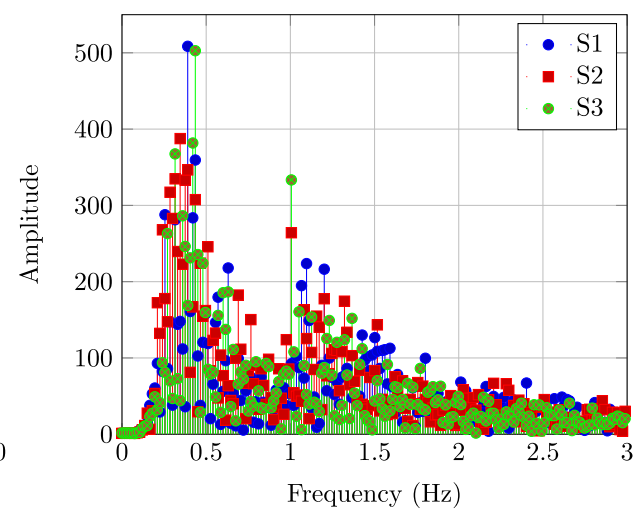

(b)
FIG. 11. Reynolds numbers for the 3 numerical results. Figure (a) shows the temporal evolution, and (b) shows the discrete Fourier analysis. 


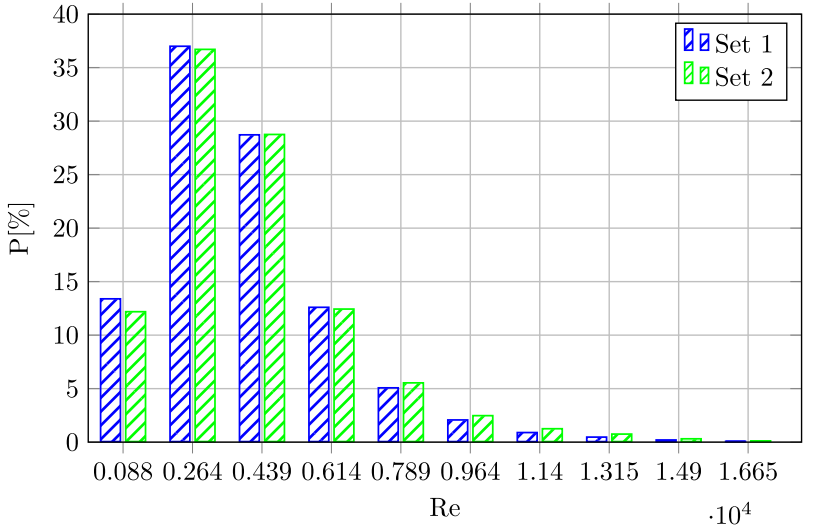

(a)

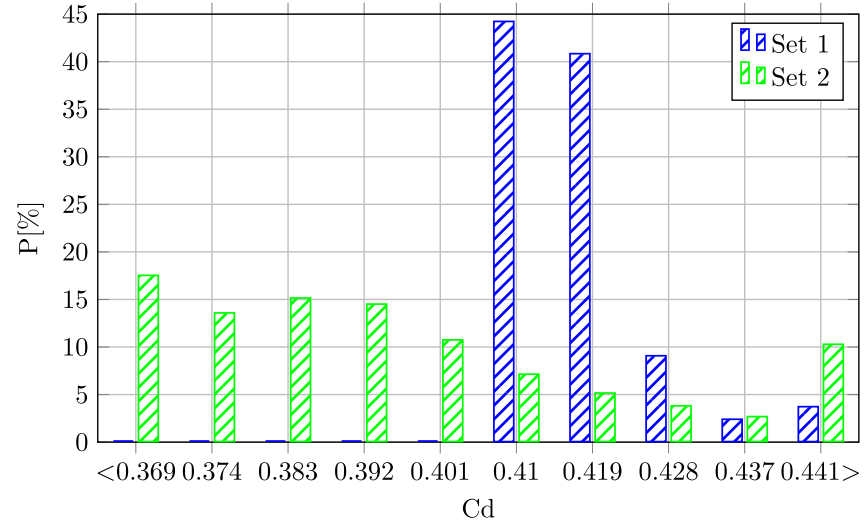

(b)

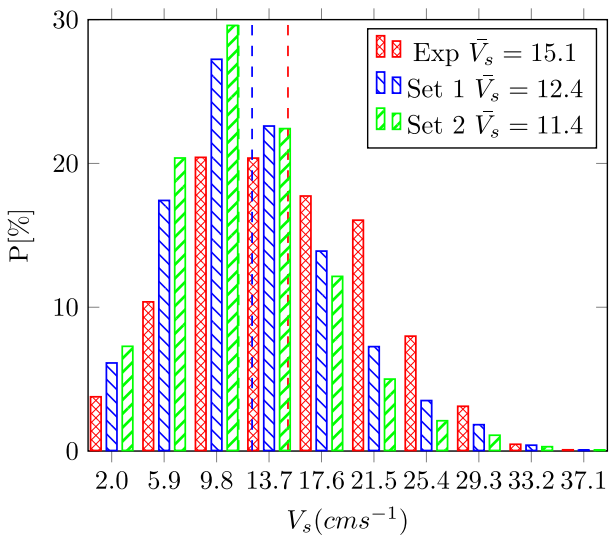

(c)

FIG. 12. Distributions: (a) Reynolds number and (b) drag coefficient from all the simulations for set 1 and set 2 , and (c) mean velocity of the sphere distribution found in the experiment and two simulation results.

Figure 12(b) shows the statistical distribution of drag coefficient $C_{D}$ using ten bins from 0.36 (minimum 0.352 ) to 0.441 , including in the last bin values that exceed the corresponding range, with a very low occurrence (maximum 5.44).

Finally, Fig. 12(c) shows the experimental and numerical, statistical distributions of the absolute (not relative to the fluid) velocity of the sphere. The experimental values are computed based on the difference of sphere positions in successive frames. The difference between the positions of the sphere at time steps corresponding to the experimental frames is used to obtain an equivalent value for the numerical simulations. The numerical values are shown for both data sets 1 and 2, corresponding to the two drag laws described in Sec. III, Eqs. (10) and (11), respectively. The velocity statistical distributions are qualitatively similar, and the difference between the mean velocities with respect to the experimental value is $17 \%$ for set 1 and $24 \%$ for set 2 . As previously mentioned, both sets differ only in the drag law used in the model, while the approximation effects near the walls are treated in the same manner.

Figure 13 shows the statistical distributions in space for both the $x$ - and $y$-axes. For instance, Fig. 13(a) shows the probability that the sphere is at a certain position $x$ of the left vessel for time instant 0 and time instant 5, by symmetry (see Fig. 3 ). The $x$ coordinate of the left vessel has been divided into 6 bins. The probabilities for the right vessel are not shown since they are equivalent to those of the left vessel via appropriate reflection symmetry. The results for time instants 3-4 are shown in Fig. 13; the total number of time instants $\left(n_{t}\right)$ is 5 . Similarly, the statistical distributions for a certain position on the $y$-axis are shown in Fig. 13(b). In all cases, the probabilities for both experimental and numerical models (see Sec. III) are shown. To quantitatively compare the two numerical drag law models with respect to the experimental values, the average absolute deviations, defined as $\|e\|=\sum_{t}\left(\frac{\sum_{i}\left|P\left(X_{i}\right)_{s}-P\left(X_{i}\right)_{e}\right|_{x}}{\operatorname{Bins}_{\mathrm{x}}}+\frac{\sum_{i}\left|P\left(X_{i}\right)_{s}-P\left(X_{i}\right)_{e}\right|_{y}}{\operatorname{Bins}_{\mathrm{y}}}\right) / n_{t}$, were computed for two different drag laws, resulting in $3.4 \%$ and $4.14 \%$ for sets 1 and 2, respectively. Although the treatment of the fluid sphere is simplified in the present model, the average absolute deviation is below $5 \%$, which indicates an acceptable error.

Magnus and Saffman forces have been computed to evaluate their influence in the present analysis. Regarding the highly 


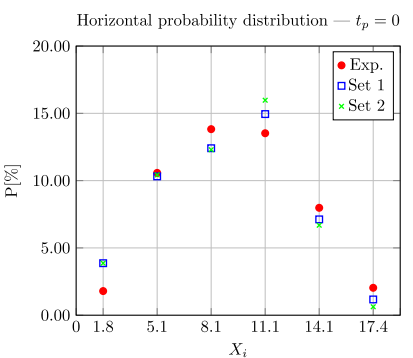

(a)

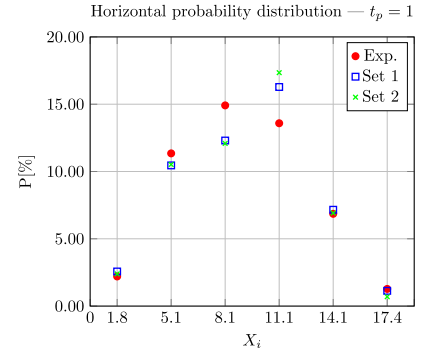

(c)

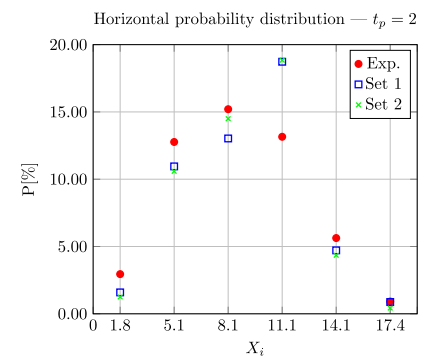

(e)

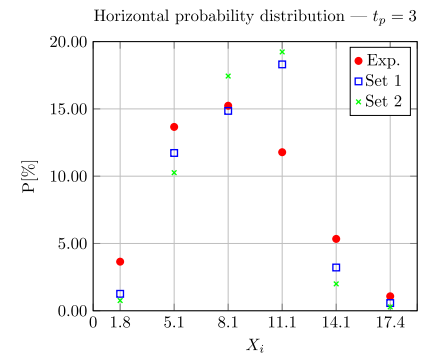

(g)

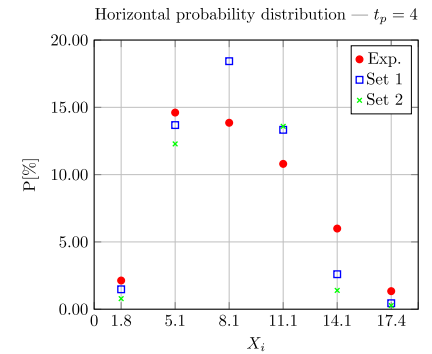

(i)

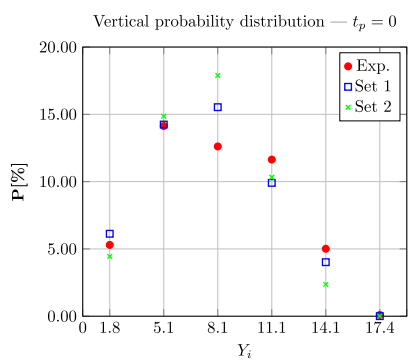

(b)

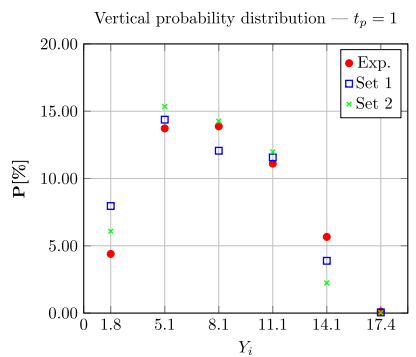

(d)

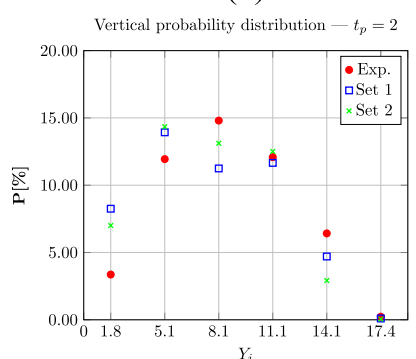

(f)

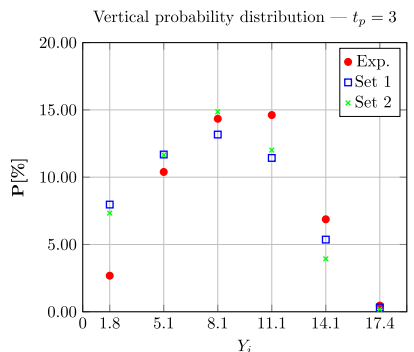

(h)

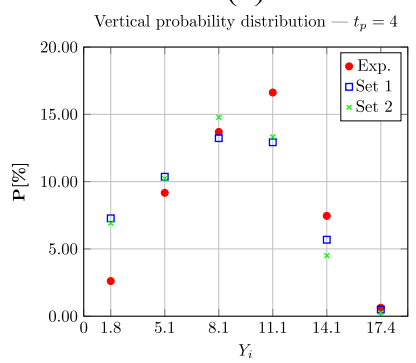

(j)
FIG. 13. Comparison of the experimental and numerical results at different instants of the analysis after symmetry reflection. Probability distributions in the $x$ (left) and $y$ (right) directions for the left vessel. transient behavior of the problem, the forces are computed using a rotation with a low recurrence of $1.1 \mathrm{rad} / \mathrm{s}$ estimated from the images. According to Ref. 46, the Magnus coefficient is taken as 0.04 ; hence, the resulting Magnus forces are bounded to $1 \%$ relative to the lift due to gradient pressure. Saffman forces evaluated as in Ref. 49 are negligible. These bounds justified not to include such forces in the present model.

\section{CONCLUSIONS}

The present work provides a numerical formulation to couple the fluid dynamics analysis of a sloshing problem with the rigid body motion of an immersed sphere. In the proposed fluid solid-rigid analyses, the fluid dynamics affect the rigid body motion; nevertheless, the sphere has no influence on the fluid dynamics. The numerical strategy involves the evaluation of the added mass, drag, lift, and wall contact forces, including boundary layer effects developed by proximity of the sphere to the wall. All these facts affect the sphere dynamics.

The proposed numerical model is tested for the settling of a single particle problem for low Reynolds numbers, and the computed results are satisfactory compared to those reported in the literature.

An experiment is proposed to validate the model in a problem with more complex dynamics. A tank, divided into two internal vessels communicated at the bottom, is mounted onto a shake table and subjected to controlled oscillating motions. The tank is filled with water, and a sphere is released inside it. The objective is to determine the position of the sphere during sloshing. The experiments are video recorded, and the sphere position is determined via the motion capturing technique.

The free surface evolution computed during the time periodic regime reproduces the experiments and provides an initial confirmation that the fluid dynamic behavior is well captured. In the present experiment, the sphere has no influence on the free surface.

The sphere dynamics are not deterministic; hence, a statistical analysis is used to evaluate the experimental and numerical data for the trajectories and velocities.

The trajectory of the sphere and its location at certain instants of the analysis are well described by the model and validated via the experiments.

The computed mean velocity of the sphere statistically approaches the experimental values.

The statistical distribution of the sphere location in space is slightly affected by the drag laws, i.e., the model satisfactorily describes the motion of the sphere. The wall effect model is described using two parameters. They could be correlated with the Reynolds number and distance to the wall to properly fit lubrication theories. This aspect was not explored in the present research and could be included in future works. Finally, the remaining discrepancies may be attributable to other facts not studied in the present analysis, e.g., strong coupling using body fitted techniques or other procedures that are out of the scope of the present analysis.

This methodology allows us to estimate the behavior of particles immersed in a viscous fluid with large displacements and is able to detect solid boundaries, in such a way that long term analyses are performed in a less costly way in comparison with immersed or embedded methods. The strategy is able to reproduce the sphere dynamics with and without fluid interfaces. 


\section{ACKNOWLEDGMENTS}

The authors are grateful for the support provided by the following research projects: Chilean Council for Scientific and Technological Research (Grant No. CONICYT-FONDECYT 1170620); the Scientific Research Projects Management Department of the Vice Presidency of Research, Development and Innovation (DICYTVRID) of Universidad de Santiago de Chile (USACH); RED CYTED 516RT0512 High Performance Computing in Engineering, Iberoamerican Scientific and Technological Development Program (CYTED, in Spanish); National Council for Scientific and Technical Research (CONICET), Argentina, Grant No. PIP-1122015-0100588; Universidad Nacional del Litoral (UNL), Argentina, Grant No. CAI+D 504-201501-00112-LI; Universidad Tecnológica Nacional (UTN), Argentina, Grant No. PID 4827; and Agencia Nacional de Promoción Científica y Tecnológica (ANPCyT), Argentina, Grant Nos. PICT-2014-2660, PICT-E-2014-0191, and PICT-2016-0640. This work was partially performed with resources from Free Software Foundation/GNU-Project, GNU/Linux OS, GNU/Octave, as well as other Open Source tools, such as Armadillo, PETSc, MPICH, LATEX, and ParaView.

\section{REFERENCES}

${ }^{1} \mathrm{~J}$. Orona, S. Zorrilla, and J. Peralta, "Sensitivity analysis using a model based on computational fluid dynamics, discrete element method and discrete phase model to study a food hydrofluidization system," J. Food Eng. 237, 183-193 (2018).

${ }^{2}$ J. Yang and F. Stern, "Sharp interface immersed-boundary/level-set method for wave-body interactions," J. Comput. Phys. 228, 6590-6616 (2009).

${ }^{3}$ A. Calderer, S. Kang, and F. Sotiropoulos, "Level set immersed boundary method for coupled simulation of air/water interaction with complex floating structures," J. Comput. Phys. 277, 201-227 (2014).

${ }^{4}$ B. Ducassou, J. Nuñez, M. Cruchaga, and S. Abadie, "A fictitious domain approach based on a viscosity penalty method to simulate wave/structure interaction," J. Hydraul. Res. 55, 847-862 (2017).

${ }^{5}$ H. H. Hu, N. Patankar, and M. Zhu, "Direct numerical simulations of fluid-solid systems using the arbitrary Lagrangian-Eulerian technique," J. Comput. Phys. 169, 427-462 (2001).

${ }^{6}$ M. A. Storti, L. Garelli, and R. R. Paz, "A finite element formulation satisfying the discrete geometric conservation law based on averaged Jacobians," Int. J. Numer. Methods Fluids 69, 1872-1890 (2012).

${ }^{7}$ A. J. Lew and G. C. Buscaglia, "A discontinuous-Galerkin-based immersed boundary method," Int. J. Numer. Methods Eng. 76, 427-454 (2008).

${ }^{8}$ R. Mittal and G. Iaccarino, "Immersed boundary methods," Annu. Rev. Fluid Mech. 37, 239-261 (2005).

${ }^{9}$ S. Tao, Z. Guo, and L.-P. Wang, "Numerical study on the sedimentation of single and multiple slippery particles in a Newtonian fluid," Powder Technol. 315, 126138 (2017).

${ }^{10} \mathrm{~S}$. Dash and T. Lee, "Two spheres sedimentation dynamics in a viscous liquid column,” Comput. Fluids 123, 218-234 (2015).

${ }^{11}$ A. Ten Cate, C. H. Nieuwstad, J. J. Derksen, and H. E. A. Van den Akker, "Particle imaging velocimetry experiments and lattice-Boltzmann simulations on a single sphere settling under gravity," Phys. Fluids 14, 4012-4025 (2002).

${ }^{12}$ C. Diaz-Goano, P. Minev, and K. Nandakumar, "A fictitious domain/finite element method for particulate flows," J. Comput. Phys. 192, 105-123 (2003).

${ }^{13}$ M. A. Cruchaga, C. M. Muñoz, and D. J. Celentano, "Simulation and experimental validation of the motion of immersed rigid bodies in viscous flows," Comput. Methods Appl. Mech. Eng. 197, 2823-2835 (2008).

${ }^{14}$ C. Choi, H. S. Yoon, and M. Y. Ha, "Flow structure around a square cylinder impacting a wall," Phys. Fluids 26, 013602 (2014).

${ }^{15}$ S. D. Costarelli, L. Garelli, M. A. Cruchaga, M. A. Storti, R. Ausensi, and S. R. Idelsohn, "An embedded strategy for the analysis of fluid structure interaction problems," Comput. Methods Appl. Mech. Eng. 300, 106-128 (2016).
${ }^{16}$ A. Pathak and M. Raessi, "A 3D, fully Eulerian, VOF-based solver to study the interaction between two fluids and moving rigid bodies using the fictitious domain method," J. Comput. Phys. 311, 87-113 (2016).

${ }^{17}$ O. M. Faltinsen, O. F. Rognebakke, and A. N. Timokha, "Transient and steadystate amplitudes of resonant three-dimensional sloshing in a square base tank with a finite fluid depth," Phys. Fluids 18, 012103 (2006).

${ }^{18}$ J. B. Frandsen, "Sloshing motions in excited tanks," J. Comput. Phys. 196, 53-87 (2004).

${ }^{19}$ O. M. Faltinsen, R. Firoozkoohi, and A. N. Timokha, "Steady-state liquid sloshing in a rectangular tank with a slat-type screen in the middle: Quasilinear modal analysis and experiments," Phys. Fluids 23, 042101 (2011).

${ }^{20}$ S. Elgeti and H. Sauerland, "Deforming fluid domains within the finite element method: Five mesh-based tracking methods in comparison," Arch. Comput. Methods Eng. 23, 323-361 (2016).

${ }^{21}$ M. Cruchaga, L. Battaglia, M. Storti, and J. D’Elía, "Numerical modeling and experimental validation of free surface flow problems," Arch. Comput. Methods Eng. 23, 139-169 (2016).

${ }^{22}$ M. A. Cruchaga, R. S. Reinoso, M. A. Storti, D. J. Celentano, and T. E. Tezduyar, "Finite element computation and experimental validation of sloshing in rectangular tanks," Comput. Mech. 52, 1301-1312 (2013).

${ }^{23}$ E. L. Grotle, H. Bihs, and V. Æsøy, "Experimental and numerical investigation of sloshing under roll excitation at shallow liquid depths," Ocean Eng. 138, 73-85 (2017).

${ }^{24}$ L. Battaglia, M. Cruchaga, M. Storti, J. D’Elía, J. Nuñez Aedo, and R. Reinoso, "Numerical modelling of 3D sloshing experiments in rectangular tanks," Appl. Math. Modell. 59, 357-378 (2018).

${ }^{25}$ P. A. Caron, M. A. Cruchaga, and A. E. Larreteguy, "Study of 3D sloshing in a vertical cylindrical tank," Phys. Fluids 30, 082112 (2018).

${ }^{26}$ P. Joshi, D. M. Escrivá, and V. Godoy, OpenCV by Example (Packt Publishing Ltd., 2016).

${ }^{27}$ G. Bueno Garcia, O. Deniz Suarez, J. L. Espinosa Aranda, J. Salido Tercero, I. Serrano Gracía, and N. Vállez Enano, Learning Image Processing with OpenCV (Packt Publishing Ltd., 2015).

${ }^{28}$ C. Sanderson and R. Curtin, "A user-friendly hybrid sparse matrix class in C++," in Mathematical Software-ICMS 2018, edited by J. H. Davenport, M. Kauers, G. Labahn, and J. Urban (Springer International Publishing, 2018), pp. 422-430.

${ }^{29}$ C. Sanderson and R. Curtin, "Armadillo: A template-based C++ library for linear algebra," J. Open Source Software 1, 26-27 (2016).

${ }^{30}$ J. C. Russ, The Image Processing Handbook (CRC Press, 2016).

${ }^{31}$ F. Kurugollu, B. Sankur, and A. E. Harmanci, "Color image segmentation using histogram multithresholding and fusion,” Image Vision Comput. 19, 915-928 (2001).

${ }^{32}$ See http://www.cimec.org.ar/petscfem for PETSc-FEM, A general purpose, parallel, multi-physics FEM program, 2019.

${ }^{33}$ A. N. Brooks and T. J. Hughes, "Streamline upwind/Petrov-Galerkin formulations for convection dominated flows with particular emphasis on the incompressible Navier-Stokes equations," Comput. Methods Appl. Mech. Eng. 32, 199-259 (1982).

${ }^{34}$ T. E. Tezduyar, "Finite elements in fluids: Stabilized formulations and moving boundaries and interfaces," Comput. Fluids 36, 191-206 (2007).

${ }^{35}$ D. Liu, W. Tang, J. Wang, H. Xue, and K. Wang, "Comparison of laminar model, RANS, LES and VLES for simulation of liquid sloshing," Appl. Ocean Res. 59, 638-649 (2016).

${ }^{36}$ P. Caron, M. Cruchaga, and A. Larreteguy, "Sensitivity analysis of finite volume simulations of a breaking dam problem," Int. J. Numer. Methods Heat Fluid Flow 25, 1718-1745 (2015).

${ }^{37}$ L. Battaglia, M. A. Storti, and J. D’Elía, “Simulation of free surface flows by a finite element interface capturing technique," Int. J. Comput. Fluid Dyn. 24, 121133 (2010).

${ }^{38}$ L. Battaglia, M. A. Storti, and J. D’Elía, “Bounded renormalization with continuous penalization for level set interface-capturing methods," Int. J. Numer. Methods Eng. 84, 830-848 (2010).

${ }^{39}$ J. Strnadel, M. Simon, and I. Machač, "Wall effects on terminal falling velocity of spherical particles moving in a Carreau model fluid," Chem. Pap. 65, 177-184 (2011). 
${ }^{40} \mathrm{H}$. Schlichting and K. Gersten, Boundary-Layer Theory (Springer, 2016).

${ }^{41} \mathrm{H}$. Brenner, "The slow motion of a sphere through a viscous fluid towards a plane surface," Chem. Eng. Sci. 16, 242-251 (1961).

${ }^{42}$ S.-Y. Lin and J.-F. Lin, "Numerical investigation of lubrication force on a spherical particle moving to a plane wall at finite Reynolds numbers," Int. J. Multiphase Flow 53, 40-53 (2013).

${ }^{43}$ C. H. Ataíde, F. A. R. Pereira, and M. A. S. Barrozo, "Wall effects on the terminal velocity of spherical particles in Newtonian and non-Newtonian fluids," Braz. J. Chem. Eng. 16, 387-394 (1999).

${ }^{44}$ A. Goldman, R. Cox, and H. Brenner, "Slow viscous motion of a sphere parallel to a plane wall-II Couette flow,” Chem. Eng. Sci. 22, 653-660 (1967).

${ }^{45} \mathrm{M}$. E. O'Neill, "A sphere in contact with a plane wall in a slow linear shear flow," Chem. Eng. Sci. 23, 1293-1298 (1968).

${ }^{46}$ N. Lukerchenko, Y. Kvurt, I. Keita, Z. Chara, and P. Vlasak, "Drag force, drag torque, and Magnus force coefficients of rotating spherical particle moving in fluid," Part. Sci. Technol. 30, 55-67 (2012).

${ }^{47}$ T. Kray, J. Franke, and W. Frank, "Magnus effect on a rotating sphere at high Reynolds numbers," J. Wind Eng. Ind. Aerodyn. 110, 1-9 (2012).

${ }^{48}$ R. Mei and J. Klausner, "Shear lift force on spherical bubbles," Int. J. Heat Fluid Flow 15, 62-65 (1994).
${ }^{49}$ A. R. Harris and C. I. Davidson, "Particle resuspension in turbulent flow: A stochastic model for individual soil grains," Aerosol Sci. Technol. 42, 613-628 (2008).

${ }^{50}$ D. Neill, D. Livelybrooks, and R. J. Donnelly, "A pendulum experiment on added mass and the principle of equivalence," Am. J. Phys. 75, 226-229 (2007).

${ }^{51}$ A. A. Kendoush, A. H. Sulaymon, and S. A. Mohammed, "Experimental evaluation of the virtual mass of two solid spheres accelerating in fluids," Exp. Therm. Fluid Sci. 31, 813-823 (2007).

${ }^{52} \mathrm{~J}$. Almedeij, "Drag coefficient of flow around a sphere: Matching asymptotically the wide trend," Powder Technol. 186, 218-223 (2008).

${ }^{53}$ F. F. Abraham, "Functional dependence of drag coefficient of a sphere on Reynolds number," Phys. Fluids 13, 2194-2195 (1970).

${ }^{54}$ F. M. White, Viscous Fluid Flow (McGraw-Hill, 1991).

${ }^{55} \mathrm{Y}$. Kim and C. S. Peskin, "A penalty immersed boundary method for a rigid body in fluid," Phys. Fluids 28, 033603 (2016).

${ }^{56} \mathrm{M}$. Rahmani and A. Wachs, "Free falling and rising of spherical and angular particles," Phys. Fluids 26, 083301 (2014).

${ }^{57}$ R. Ortega, J. C. García Orden, M. Cruchaga, and C. García, "Energy-consistent simulation of frictional contact in rigid multibody systems using implicit surfaces and penalty method," Multibody Syst. Dyn. 41, 275-295 (2017). 